\title{
Geranylgeranylacetone protects against morphine-induced hepatic and renal damage in mice
}

\author{
FU-CHENG LUO ${ }^{1}$, LU ZHAO ${ }^{1}$, JUAN DENG ${ }^{1}$, MIN LIANG $^{1}$, XIAN-SI ZENG ${ }^{1}$, HUA LIU ${ }^{2}$ and JIE BAI $^{1}$ \\ ${ }^{1}$ Medical Faculty, Kunming University of Science and Technology, Kunming 650500; \\ ${ }^{2}$ Department of Cadre Health Protection, The First People's Hospital of Yunnan Province, Kunming 650032, P.R. China
}

Received July 17, 2012; Accepted November 21, 2012

DOI: $10.3892 / \mathrm{mmr} .2012 .1217$

\begin{abstract}
The acute or chronic administration of opioid drugs may induce oxidative damage and cellular apoptosis in the liver and kidney, and hence result in hepatic and renal damage. Thioredoxin-1 (Trx-1) and heat shock protein 70 (Hsp70) are emerging as important modulators of cellular functions. They have been shown to be involved in cellular protective mechanisms against a variety of toxic stressors. The present study was designed to investigate the effects of geranylgeranylacetone (GGA), a pharmacological inducer of Trx-1 and Hsp70, on morphine-induced hepatic and renal damage. Morphine induced apoptosis in the liver and kidney through the mitochondria-mediated apoptosis pathway, but not the endoplasmic reticulum-mediated pathway. The activation of caspases- 9 and -3 was attenuated by pre-treatment with GGA. In addition, the morphine-induced increase of malondialdehyde (MDA) levels was suppressed by GGA. Furthermore, GGA enhanced morphine-induced expression of Trx-1 and Hsp70 in the liver and kidney. The findings of this study suggest that GGA may be a safe and novel therapeutic agent for morphine-induced hepatic and renal damage.
\end{abstract}

\section{Introduction}

Addiction to opioid drugs such as morphine is a universal social and public problem. The repeated administration of opioid drugs may lead to the development of analgesic tolerance and dependence, which limit the clinical use of opioid drugs in pain treatment (1). Furthermore, prolonged exposure to opioid drugs may result in pathological changes in the liver in almost $100 \%$ of cases $(2,3)$. A previous study found that $44 \%$ of drug addicts suffered from acute hepatitis, $34 \%$ from chronic hepatitis and 16\% from liver damage (4). Lately, clinical studies also suggest that opiate addicts are at increased risk of progressive renal failure (5-7). Renal diseases in opiate

Correspondence to: Dr Jie Bai, Medical Faculty, Kunming University of Science and Technology, Kunming 650500, P.R. China E-mail: jiebai662001@yahoo.com.cn

Key words: geranylgeranylacetone, morphine, liver, kidney, thioredoxin-1, heat shock protein 70 addicts are associated with nephritic syndrome, acute glomerulonephritis and interstitial nephritis $(8,9)$. The acute or chronic administration of opioid drugs may induce oxidative damage and cellular apoptosis in the liver and kidney, and hence result in hepatic and renal damage (10-13). Considering the role of the liver and kidney in metabolism and detoxification, opioid drug-induced liver and kidney toxicity has been a crucial issue in the therapy for drug abuse.

Previous studies have shown that blocking oxidative damage is a useful strategy for the treatment of opioid druginduced hepatic and renal damage (14). There are several molecules to counteract oxidative stress in the body, such as thioredoxin-1 (Trx-1) and heat shock protein 70 (Hsp70). Trx-1 is a small ubiquitous protein with redox-controlled cell functions $(15,16)$. Trx-1 has radical-scavenging activity and couples with peroxiredoxin to scavenge hydrogen peroxide $(17,18)$. Trx-1 is able to protect cells from hydrogen peroxide, UV irradiation and ischemic reperfusion $(19,20)$. Hsp70 is a stress-inducible protein that prevents protein aggregation and facilitates refolding of dysfunctional proteins (21). Hsp70 is able to enhance the survival of cells and inhibits apoptosis induced by oxidative damage during various stress conditions. Geranylgeranylacetone (GGA) is an acyclic polyisoprenoid and widely used as an anti-ulcer drug in the clinical setting. Previous studies have suggested that GGA induced Trx-1 and Hsp70 expression in various cells to exert cytoprotection (22-24). GGA has been widely reported to exert cytoprotective effect against various stresses, such as oxidative stress in the gastric mucosa, liver, heart and brain (25-28). Our previous study also has demonstrated that GGA protected against morphine-induced hyperlocomotion, rewarding effect and withdrawal syndrome in mice (29). However, there are no studies of the role of GGA in the liver and kidney toxicity induced by opioid drugs. In the present study, we postulated that GGA induces Trx-1 and Hsp70 expression, which may in turn prevent opiate-induced hepatic and renal injury. The present study was designed to evaluate the protective effect of GGA against morphine-induced hepatic and renal damage in mice.

\section{Materials and methods}

Reagents. GGA was purchased from Eisai (Tokyo, Japan). Anti-mouse Trx-1 rabbit polyclonal antibody was obtained from Redox Bioscience (Kyoto, Japan). Antibodies 
(pro-caspase-12 and -9, caspase-3, $\beta$-actin and Hsp70) were purchased from Santa Cruz Biotechnology, Inc. (Santa Cruz, CA, USA). Morphine hydrochloride was purchased from the Shenyang First Pharmaceutical Factory, Northeast Pharmaceutical Group Corporation (Shenyang, China). Mouse malondialdehyde (MDA) enzyme-linked immunosorbent assay (ELISA) kit was purchased from R\&D Systems (Minneapolis, MN, USA).

Animals. Sixteen C57BL/6 mice (Chongqing Medical University, China; age, 6-7 weeks) were used in the experiments. The mice were housed in plastic cages and kept on a 12-h light-dark cycle and had free access to food and water. The animals were cared for and used in accordance with the National Institutes of Health Guide for the Care and Use of Laboratory Animals. The experiment was approved by the local Committee on Animal Use and Protection.

GGA and morphine treatment. Granulated GGA was suspended in saline immediately before use. Mice were administered saline or GGA ( $800 \mathrm{mg} / \mathrm{kg}$, orally), using a micropipette $2 \mathrm{~h}$ prior to morphine treatment. The mouse model of morphine dependence was created as described in our previous study (29). The mice were injected with escalating morphine doses twice daily $(10,20,40,60,80$ and $100 \mathrm{mg} / \mathrm{kg}$ ) for 7 days. Two hours following the last morphine injection, the mice were sacrificed using deep anesthesia, and then heart perfusion was performed using saline. The liver and kidney tissues were rapidly dissected, immediately frozen and stored in a deep freezer at $-80^{\circ} \mathrm{C}$ until use.

Western blot analysis. Protein lysates were prepared using a solubilizing solution [20 mM Tris- $\mathrm{HCl}(\mathrm{pH} 7.4), 150 \mathrm{mM}$ $\mathrm{NaCl}, 1 \%$ NP-40, 1 mM EDTA, 1 mM PMSF, 1 mM EGTA, $1 \%$ Triton $\mathrm{X}-100,2.5 \mathrm{mM}$ sodium pyrophosphate, $1 \mathrm{mM}$ $\mathrm{Na}_{3} \mathrm{VO}_{4}, 1 \mathrm{mM} \beta$-glycerolphosphate and $1 \mathrm{mg} / \mathrm{ml}$ leupeptin]. Protein concentration was determined using the Bio-Rad protein assay reagent (Bio-Rad, Hercules, CA, USA). An equal quantity of proteins was separated by $10 \%$ (for Hsp70), $12 \%$ (for pro-caspase-12) or 15\% (for pro-caspase-9, caspase-3 and Trx-1) SDS-PAGE and transferred to a PVDF membrane (Millipore Corporation, Billerica, MA, USA). The membrane was soaked in $10 \%$ skimmed milk (in PBS, pH 7.2, containing $0.1 \%$ Tween-20) overnight at $4{ }^{\circ} \mathrm{C}$, then incubated with primary antibody followed by peroxidase-conjugated anti-mouse or anti-rabbit IgG (KPL, Gaithersburg, MD, USA). The epitope was visualized using an ECL Western Blot Detection kit (Millipore Corporation). Densitometry analysis was performed using the ImageJ software.

ELISA. The liver and kidney tissues were pooled and homogenized in PBS. Samples were spun at 4,000 rpm for $15 \mathrm{~min}$ at $4^{\circ} \mathrm{C}$. The supernatant was aliquoted and stored at $-80^{\circ} \mathrm{C}$ for future study. The concentration of MDA was measured using specific ELISA kits (R\&D, USA), according to the manufacturers' instructions.

Statistical analysis. Data are expressed as the mean \pm SD values. Statistical analysis was performed using the SPSS software. The one-way ANOVA followed by a post hoc
A

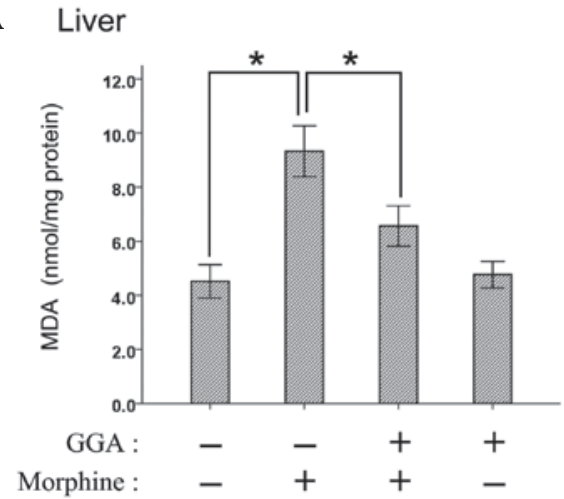

B

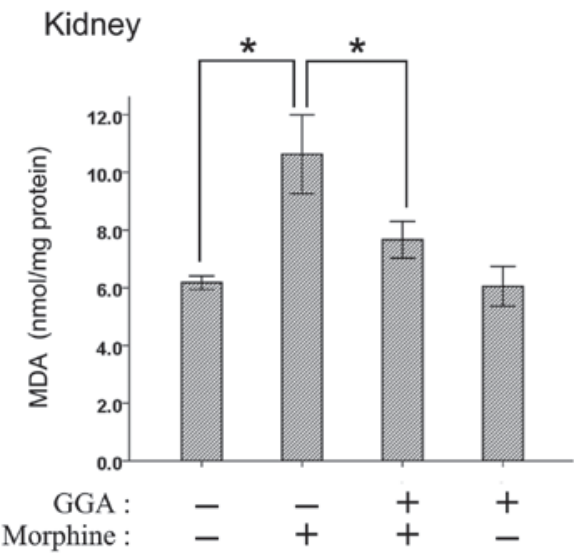

Figure 1. GGA suppressed morphine-induced lipid peroxidation in the liver and kidney. Mice were injected with escalating morphine doses twice daily $(10,20,40,60,80$ and $100 \mathrm{mg} / \mathrm{kg}), 2 \mathrm{~h}$ after GGA treatment $(800 \mathrm{mg} / \mathrm{kg}$, orally) for 7 days. Two hours after the last morphine injection, the liver and kidney tissues of mice were dissected. The MDA level in (A) the liver and (B) kidney was measured using ELISA. Values are the mean \pm SD; $n=4$. Asterisks indicate a statistically significant difference ( $\mathrm{P}<0.05)$. GGA, geranylgeranylacetone; MDA, malondialdehyde; ELISA, enzyme-linked immunosorbent assay.

multiple comparison test was used to compare control and treated groups. $\mathrm{P}<0.05$ was considered to indicate a statistically significant difference.

\section{Results}

Effect of GGA on lipid peroxide formation in the liver and kidney after chronic morphine treatment. As an important marker of oxidative lipid damage, MDA was examined to estimate the extent of lipid peroxidation in the present study. The levels of MDA in the liver and kidney were significantly increased after chronic morphine treatment (Fig. 1). By contrast, the pre-treatment of GGA significantly reduced lipid peroxide content in the liver and kidney.

Effect of GGA on morphine-induced apoptosis in the liver and kidney. To clarify the manner in which GGA affects cell death pathways involved in the morphine-induced hepatic and renal damage, the alterations of pro-caspases-9 and -12 and caspase-3 were examined following chronic morphine administration with or without GGA pre-treatment using western blotting. Chronic morphine administration led to the activa- 

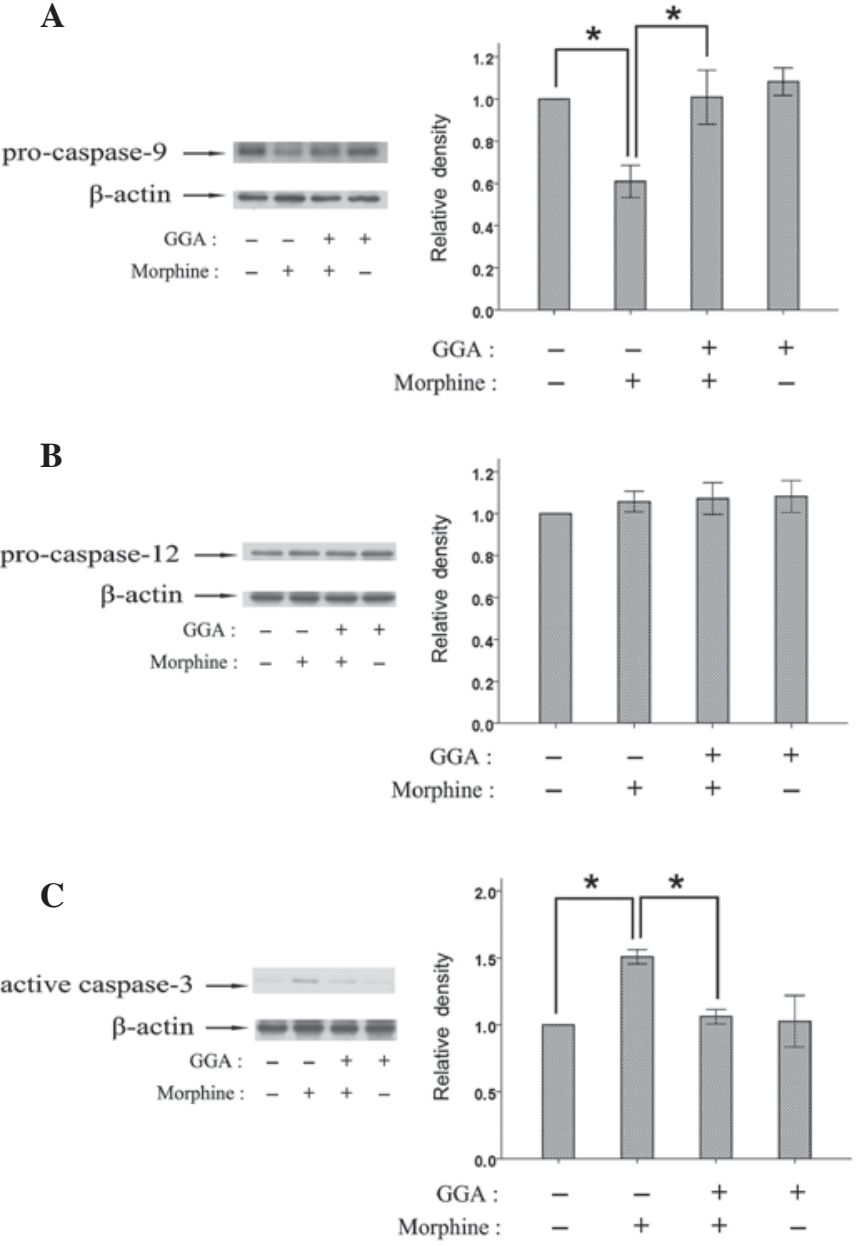

Figure 2. GGA regulated the activation of caspases induced by morphine in the liver. Mice were injected with escalating morphine doses twice daily (10, $20,40,60,80$ and $100 \mathrm{mg} / \mathrm{kg}), 2 \mathrm{~h}$ after GGA treatment $(800 \mathrm{mg} / \mathrm{kg}$, orally) for 7 days. Two hours after the last morphine injection, the liver tissues of mice were dissected, and the expression of (A) pro-caspase-9, (B) -12 and (C) caspase-3 was detected using western blotting. Quantification is shown in the right panels (error bars represent the mean $\pm S D ; n=4$ ). Density values for each band were normalized first to loading control and then to the control group. Asterisks indicate a statistically significant difference ( $\mathrm{P}<0.05)$. GGA, geranylgeranylacetone.

tion of caspases- 9 and -3 in the liver and kidney, while the level of pro-capase-12 was not affected by morphine (Figs. 2 and 3). Furthermore, the pre-treatment with GGA significantly inhibited the activation of caspases- 9 and -3 induced by morphine in the liver and kidney.

Effects of GGA on Trx-1 and Hsp70 expression in the liver and kidney following chronic morphine treatment. To investigate the roles of Trx-1 and Hsp70 underlying the protective effect of GGA against morphine-induced hepatic and renal damage, the levels of Trx-1 and Hsp70 were examined following morphine treatment. Morphine significantly increased the levels of Trx-1 and Hsp70 expression in the liver and kidney (Figs. 4 and 5). GGA was also found to enhance morphine-induced expression of Trx-1 and Hsp70 in the liver and kidney. The above data indicated that GGA induced the expression of Trx-1 and Hsp70, which may be involved in the protective roles of GGA against morphine-induced hepatic and renal damage.
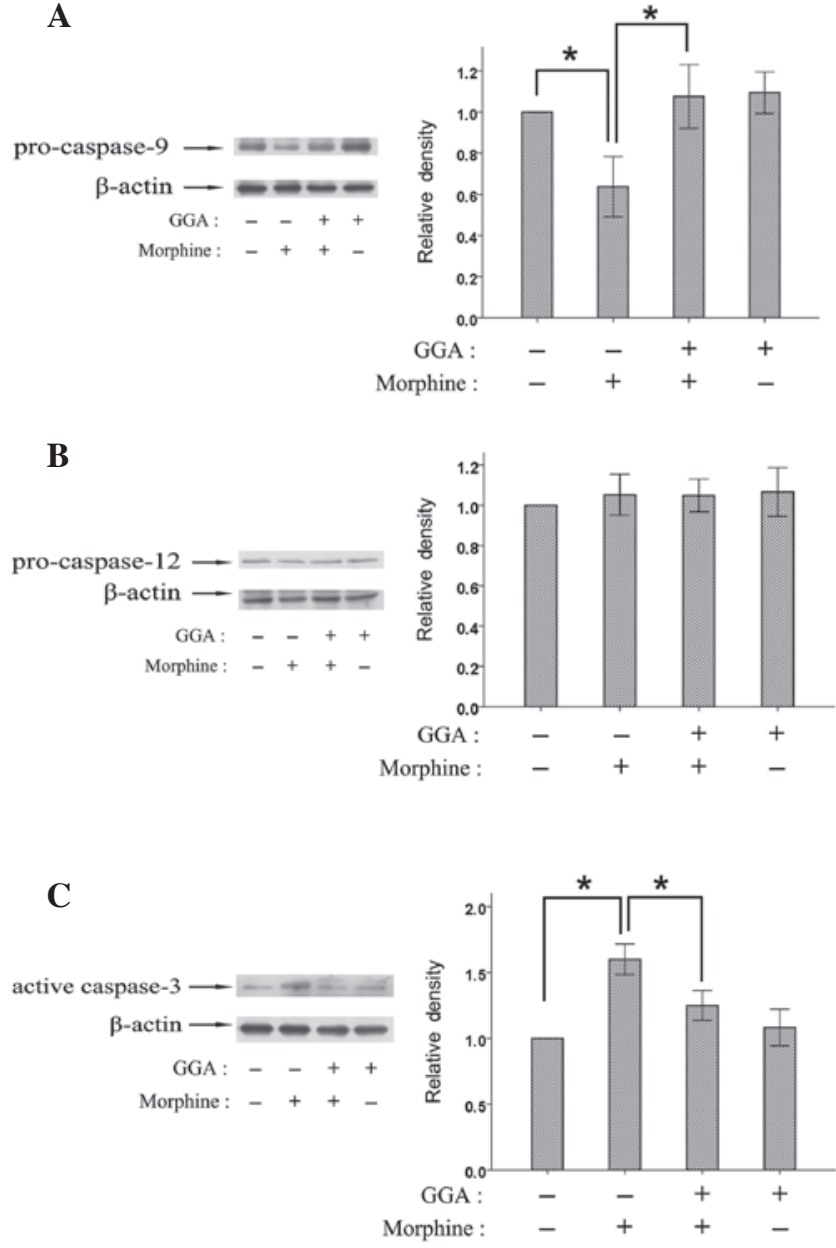

Figure 3. GGA regulated the activation of caspases induced by morphine in the kidney. Mice were injected with escalating morphine doses twice daily $(10,20,40,60,80$ and $100 \mathrm{mg} / \mathrm{kg}), 2 \mathrm{~h}$ after GGA treatment $(800 \mathrm{mg} / \mathrm{kg}$, orally) for 7 days. Two hours after the last morphine injection, the kidney tissues of mice were dissected, and the expression of (A) pro-caspase-9, (B) -12 and $(C)$ caspase- 3 was detected using western blotting. Quantification is shown in the right panels (error bars represent the mean $\pm S D ; n=4$ ). Density values for each band were normalized first to loading control and then to the control group. Asterisks indicate a statistically significant difference $\left({ }^{*} \mathrm{P}<0.05\right)$. GGA, geranylgeranylacetone.

\section{Discussion}

GGA is an anti-ulcer drug developed in Japan and is known to protect against organ and cell damage via inducing Hsp70 or Trx-1 expression. In the present study, we found that the pre-treatment of GGA suppressed morphine-induced lipid peroxidation and activation of caspases- 9 and -3 in the liver and kidney. These findings are consistent with those of previous studies, which reported the protective effect of GGA against hepatic and renal damage caused by other stimulations $(30,31)$. GGA suppressed the $\mathrm{H}_{2} \mathrm{O}_{2}$ and ethanol-induced activation of JNK and caspases-9 and -3 , leading to significant inhibition of apoptosis in rat hepatocytes (32). Oral administration of GGA protects liver damage following massive hepatectomy, warm ischemic-reperfusion injury and exposure of acetaminophen in rats $(31,33,34)$. In addition, GGA ameliorated ischemic acute renal failure via inducing Hsp70 expression (35). The dose of GGA used in this study is $800 \mathrm{mg} / \mathrm{kg}$. However, it did not show toxic effects in the present study. The results of our 
A
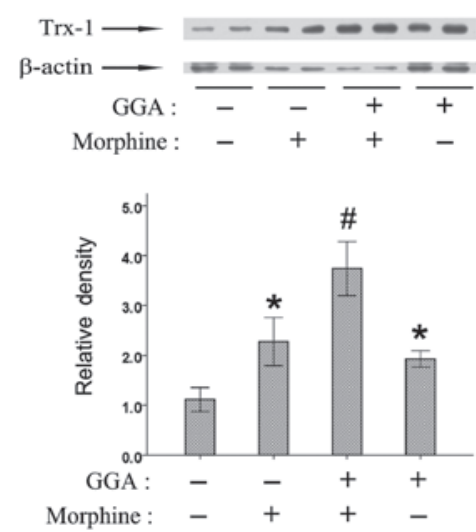

B

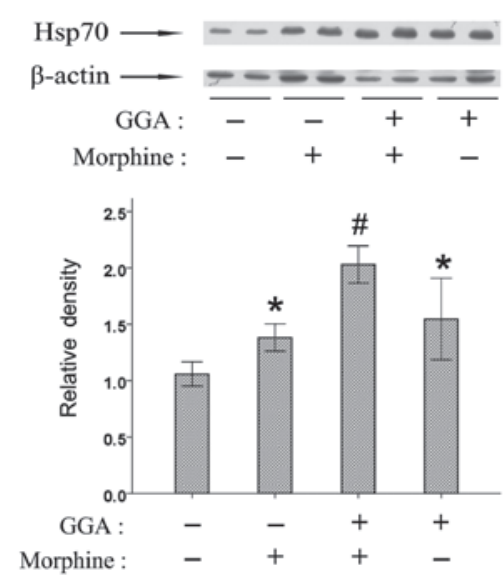

Figure 4. Changes in the expression of Trx-1 and Hsp70 after morphine treatment in the liver. Mice were injected with escalating morphine doses twice daily $(10,20,40,60,80$ and $100 \mathrm{mg} / \mathrm{kg}), 2 \mathrm{~h}$ after GGA treatment $(800 \mathrm{mg} / \mathrm{kg}$, orally) for 7 days. Two hours after the last morphine injection, the liver tissues of mice were dissected, and the expression of (A) Trx-1 and (B) Hsp70 was detected using western blotting. Quantification is shown in the right panels (error bars represent the mean $\pm S D ; n=4$ ). Density values for each band were normalized first to loading control and then to the control group. ${ }^{*} \mathrm{P}<0.05$ vs. the control group, and ${ }^{\#} \mathrm{P}<0.05$ vs. the chronic morphine-treated group. GGA, geranylgeranylacetone; Hsp, heat shock protein.

previous animal experiment revealed that the pre-treatment of GGA ( $800 \mathrm{mg} / \mathrm{kg}, 1$ week) protected against morphineinduced hyperlocomotion, rewarding effect and withdrawal syndrome (29). Oral GGA (600 and 1,200 $\mathrm{mg} / \mathrm{kg}, 7$ weeks) ameliorates symptomatic phenotypes of spinal and bulbar muscular atrophy mice (36). There is little information concerning any adverse drug effects of GGA. These results suggest that GGA is a promising safe therapeutic candidate for opioid drug-induced hepatic and renal damage. Besides the cytoprotection in the liver and kidney, GGA also exerts protective effects against various stresses in other organs, including the heart and brain (37-39). The toxicities of morphine in a number of tissues, including the spinal cord and brain, have also been reported $(40,41)$. Whether or not GGA is able to protect against morphine-induced toxicity in other organs remains to be elucidated.

Liver and kidney toxicities have been reported in chronic use of morphine $(10,42)$. Serum aspartate aminotransferase and alanine aminotransferase levels are increased following exposure to morphine in rats (13). Animal studies have
A
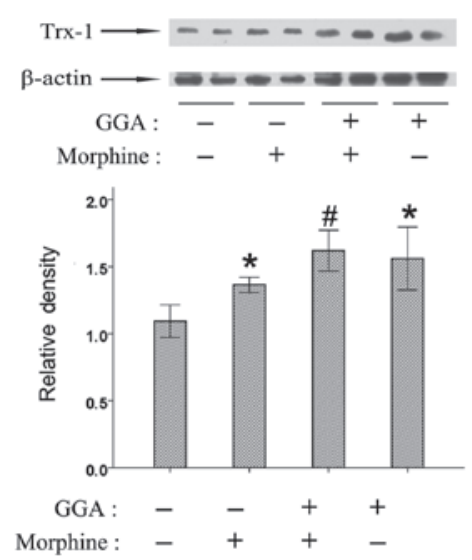

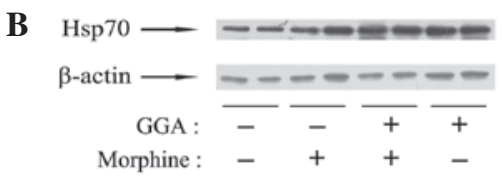

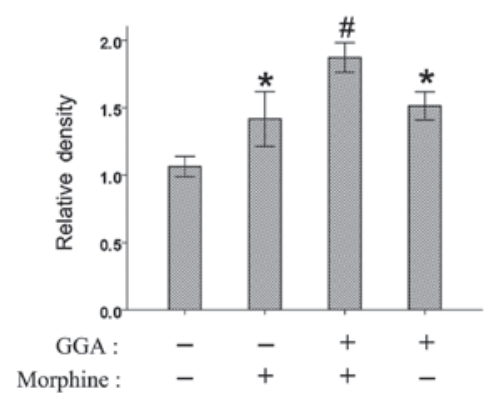

Figure 5. Changes in the expression of Trx-1 and Hsp70 after morphine treatment in the kidney. Mice were injected with escalating morphine doses twice daily $(10,20,40,60,80$ and $100 \mathrm{mg} / \mathrm{kg}), 2 \mathrm{~h}$ after GGA treatment ( $800 \mathrm{mg} / \mathrm{kg}$, orally) for 7 days. Two hours after the last morphine injection, the kidney tissues of mice were dissected, and the expression of (A) Trx-1 and (B) Hsp70 was detected using western blotting. Quantification is shown in the right panels (error bars represent the mean $\pm S D ; n=4$ ). Density values for each band were normalized first to loading control and then to the control group. ${ }^{*} \mathrm{P}<0.05$ vs. the control group, and ${ }^{\#} \mathrm{P}<0.05$ vs. the chronic morphinetreated group. GGA, geranylgeranylacetone; Hsp, heat shock protein.

shown that morphine may have a direct effect on the glomerulus, causing oxidative stress, proliferation of fibroblasts and a decrease in the degradation of type IV collagen (43-45). Excessive free radical formation or antioxidant deficiency may result in oxidative stress, a possible mechanism of the toxicity of opioid drugs. Heroin abusers exhibited increased levels of lipoperoxides and nitric oxide. The levels of various antioxidants, including superoxide dismutase, glutathione and catalase, were decreased following heroin or morphine exposure (46). It has also been reported that morphine may result in lipid peroxidation in the liver and kidney (13). In the present study, we found that morphine increased MDA levels, demonstrating lipid peroxidation in the liver and kidney following chronic morphine treatment.

Extrinsic and intrinsic cell death pathways have been shown to be involved in morphine-induced apoptosis. The extrinsic cell death pathway has been reported to contribute to morphine-induced macrophage apoptosis. Morphine enhanced Fas and FasL expression in macrophages (47). The intrinsic cell death pathway includes the mitochondria-mediated 
apoptosis pathway and endoplasmic reticulum (ER)-mediated apoptosis pathway. Although activation of several distinct upstream pathways may lead to apoptosis, caspase- 3 may be a common integration point (48). In the present study, we found that caspase- 3 was activated by morphine, suggesting that morphine leads to apoptosis in the liver and kidney. Mitochondrial dysfunction leads to the release of cytochrome $\mathrm{c}$ into the cytoplasm, and initiates the activation of the caspase cascade by triggering the activation of caspase- 9 , which elicits the activation of caspase-3, leading to the morphological changes associated with apoptosis (49). The results of in vivo and in vitro studies indicate that caspases- 9 and -3 are activated following morphine treatment in macrophages, the spinal cord or brain $(41,50)$. In this study, we demonstrated that morphine decreased the level of pro-caspase- 9 expression, indicating that morphine induces apoptosis through the mitochondriamediated pathway in the liver and kidney. Pro-caspase-12 is localized on the cytoplasmic side of ER and is proteolytically activated by excess ER stress. ER stress-induced cell death has been reported to be mediated by the activation of caspase-12 (51). Caspase-12-deficient mice are resistant to ER stress-induced apoptosis (52). However, in the present study, the level of pro-caspase-12 expression was not affected by morphine in the liver and kidney. These findings suggest that the ER-mediated apoptosis pathway is not involved in the morphine-induced apoptosis in the liver and kidney.

Eukaryotic cells have developed multiple mechanisms to mount a response against environmental stressors. Cellular stress responses require the activation of pro-survival pathways and the production of molecules with antioxidant and anti-apoptotic activities, allowing cells to effectively counteract diverse stresses. Among the cellular pathways providing protection against environmental stressors, a key role is played by vitagenes, such as heat shock proteins and Trx-1 (53). Trx-1 expression is upregulated by various stressors, including viral infection, mitogens, polycyclic aromatic hydrocarbons, $\mathrm{X}$-ray and UV irradiation, hydrogen peroxide and ischemic reperfusion (54). Hsp70 is also induced in response to a wide variety of stressors, such as heat shock, ischemia, hydrogen peroxide and light damage (26). The induction of Trx-1 and Hsp70 contributes to cytoprotection against diverse stresses. It has been reported that morphine increased Hsp70 expression in the frontal cortex, amygdale and spinal cord $(41,55,56)$. Our previous study demonstrated that the levels of Trx-1 and Hsp70 expression in the nucleus accumbens of mice brain are increased after chronic morphine treatment (29). In the present study, we found that Trx-1 and Hsp70 were induced by morphine in the liver and kidney. These results suggest that cellular defense mechanisms are activated in the liver and kidney to resist the cellular damage caused by morphine.

Modulation of endogenous cellular defense mechanisms via the stress response signaling is becoming an innovative approach to therapeutic intervention in diseases. Several studies have used Trx-1 or Hsp70 inducers, including GGA, sulforaphane, panaxatriol saponins and neurotropin, for therapeutic purposes (57-60). In the present study, we found that the increased level of MDA in the liver and kidney was suppressed by the pre-treatment with GGA. The morphineinduced decrease of pro-caspase- 9 was suppressed by GGA. Furthermore, GGA inhibited the morphine-induced activation of caspase- 3 in the liver and kidney. These results suggest that GGA treatment may be beneficial for preventing morphineinduced hepatic and renal damage. In the present study, we also demonstrated that GGA increased Trx-1 and Hsp70 expression in the liver and kidney following morphine treatment. A previous study has shown that morphine promotes p38 MAPK phosphorylation via opiate receptors through TGF- $\beta$ and iNOS-mediated downstream signaling (47). Trx-1 is known to have radical-scavenging activity and scavenge hydrogen peroxide in cooperation with peroxiredoxin. In addition, Trx-1 has been reported to act as an endogenous inhibitor of apoptosis signaling kinase 1 and p38 MAPK $(61,62)$. Hsp70 regulates the cellular redox status by modulating glutathionerelated enzyme activities (63). Hsp70 enhances the survival of cells and prevents apoptosis against oxidative damage during various stress conditions (21). The antioxidant effects of GGA were previously reported in the ethanol- and $\mathrm{H}_{2} \mathrm{O}_{2}$-induced cultured rat hepatocyte injury and in light-induced photoreceptor cell damage through inducing Trx-1 expression $(58,64)$. Thus, the protective effects of GGA against morphine-induced lipid peroxidation and apoptosis in the liver and kidney might contribute to the increased expression of Trx-1 and Hsp70.

In conclusion, the findings of the present study indicate that morphine induces lipid peroxidation and mitochondria-mediated apoptosis, and regulates Trx-1 and Hsp70 expression in the liver and kidney. GGA induced Trx-1 and Hsp70 expression and suppressed morphine-induced lipid peroxidation and apoptosis in the liver and kidney. Our results suggest that GGA is a promising therapeutic candidate for morphine-induced hepatic and renal damage.

\section{Acknowledgements}

This study was supported by the National Natural Science Foundation of China (no. 81160162,U1202227), a grant from the Candidates of the Young and Middle Aged Academic Leaders of Yunnan Province (no. 2006PY01-07), Application Basic Research Fund of the Yunnan Province (no. 2009ZC165M), the foundation of Excellent Doctor Degree Dissertation of the Kunming University of Science and Technology and grant from the Key Laboratory of Medical Neurobiology, Kunming University of Science and Technology, China.

\section{References}

1. Christie MJ: Cellular neuroadaptations to chronic opioids: tolerance, withdrawal and addiction. Br J Pharmacol 154: 384-396, 2008.

2. Weller IV, Cohn D, Sierralta A, et al: Clinical, biochemical, serological, histological and ultrastructural features of liver disease in drug abusers. Gut 25: 417-423, 1984.

3. Colombo JP and Colombo J: Plasma gamma-glutamyl transpeptidase in heroin addicts. Clin Chim Acta 95: 483-486, 1979.

4. May B and Helmstaedt D: Liver disease in drug addicts: clinical course-toxicological and clinical pharmacological aspects. Int J Clin Pharmacol Biopharm 12: 50-56, 1975.

5. Cunningham EE, Brentjens JR, Zielezny MA, Andres GA and Venuto RC: Heroin nephropathy. A clinicopathologic and epidemiologic study. Am J Med 68: 47-53, 1980.

6. Connolly JO, Gillmore JD, Lachmann HJ, Davenport A, Hawkins PN and Woolfson RG: Renal amyloidosis in intravenous drug users. QJM 99: 737-742, 2006.

7. Dettmeyer R, Wessling B and Madea B: Heroin associated nephropathy - a post-mortem study. Forensic Sci Int 95: 109-116, 1998. 
8. Bakir AA and Dunea G: Drugs of abuse and renal disease. Curr Opin Nephrol Hypertens 5: 122-126, 1996.

9. Jaffe JA and Kimmel PL: Chronic nephropathies of cocaine and heroin abuse: a critical review. Clin J Am Soc Nephrol 1: 655-667, 2006

10. Payabvash S, Beheshtian A, Salmasi AH, et al: Chronic morphine treatment induces oxidant and apoptotic damage in the mice liver. Life Sci 79: 972-980, 2006.

11. Panchenko LF, Pirozhkov SV, Nadezhdin AV, Baronets Vlu and Usmanova NN: Lipid peroxidation, peroxyl radical-scavenging system of plasma and liver and heart pathology in adolescence heroin users. Vopr Med Khim 45: 501-506, 1999 (In Russian).

12. Sumathi T and Niranjali Devaraj S: Effect of Bacopa monniera on liver and kidney toxicity in chronic use of opioids. Phytomedicine 16: 897-903, 2009.

13. Atici S, Cinel I, Cinel L, Doruk N, Eskandari G and Oral U: Liver and kidney toxicity in chronic use of opioids: an experimental long term treatment model. J Biosci 30: 245-252, 2005.

14. Zhang YT, Zheng QS, Pan J and Zheng RL: Oxidative damage of biomolecules in mouse liver induced by morphine and protected by antioxidants. Basic Clin Pharmacol Toxicol 95: 53-58, 2004.

15. Burke-Gaffney A, Callister ME and Nakamura H: Thioredoxin: friend or foe in human disease? Trends Pharmacol Sci 26 : 398-404, 2005

16. Lillig $\mathrm{CH}$ and Holmgren A: Thioredoxin and related molecules - from biology to health and disease. Antioxid Redox Signal 9: 25-47, 2007.

17. Chae HZ, Chung SJ and Rhee SG: Thioredoxin-dependent peroxide reductase from yeast. J Biol Chem 269: 27670-27678, 1994.

18. Das KC and Das CK: Thioredoxin, a singlet oxygen quencher and hydroxyl radical scavenger: redox independent functions. Biochem Biophys Res Commun 277: 443-447, 2000.

19. Nakamura H, Matsuda M, Furuke K, et al: Adult T cell leukemia-derived factor/human thioredoxin protects endothelial F-2 cell injury caused by activated neutrophils or hydrogen peroxide. Immunol Lett 42: 75-80, 1994.

20. Takagi Y, Mitsui A, Nishiyama A, et al: Overexpression of thioredoxin in transgenic mice attenuates focal ischemic brain damage. Proc Natl Acad Sci USA 96: 4131-4136, 1999.

21. Evans CG, Chang L and Gestwicki JE: Heat shock protein 70 (hsp70) as an emerging drug target. J Med Chem 53: 4585-4602, 2010.

22. Bai J, Nakamura H, Hattori I, Tanito $M$ and Yodoi J: Thioredoxin suppresses 1-methyl-4-phenylpyridinium-induced neurotoxicity in rat PC12 cells. Neurosci Lett 321: 81-84, 2002.

23. Dekigai H, Nakamura H, Bai J, et al: Geranylgeranylacetone promotes induction and secretion of thioredoxin in gastric mucosal cells and peripheral blood lymphocytes. Free Radic Res 35: 23-30, 2001.

24. Fudaba Y, Ohdan H, Tashiro H, et al: Geranylgeranylacetone, a heat shock protein inducer, prevents primary graft nonfunction in rat liver transplantation. Transplantation 72: 184-189, 2001.

25. Yamagami K, Yamamoto $\mathrm{Y}$, Ishikawa Y, Yonezawa K, Toyokuni S and Yamaoka Y: Effects of geranyl-geranyl-acetone administration before heat shock preconditioning for conferring tolerance against ischemia-reperfusion injury in rat livers. J Lab Clin Med 135: 465-475, 2000.

26. Ooie T, Takahashi N, Saikawa T, et al: Single oral dose of geranylgeranylacetone induces heat-shock protein 72 and renders protection against ischemia/reperfusion injury in rat heart. Circulation 104: 1837-1843, 2001.

27. Fujiki M, Kobayashi H, Abe T and Ishii K: Astroglial activation accompanies heat shock protein upregulation in rat brain following single oral dose of geranylgeranylacetone. Brain Res 991: 254-257, 2003.

28. Yamanaka K, Takahashi N, Ooie T, Kaneda K, Yoshimatsu H and Saikawa T: Role of protein kinase $\mathrm{C}$ in geranylgeranylacetone-induced expression of heat-shock protein 72 and cardioprotection in the rat heart. J Mol Cell Cardiol 35: 785-794, 2003.

29. Luo FC, Qi L, Lv T, et al: Geranylgeranylacetone protects mice against morphine-induced hyperlocomotion, rewarding effect, and withdrawal syndrome. Free Radic Biol Med 52: 1218-1227, 2012.

30. Mao H, Li Z, Zhou Y, et al: HSP72 attenuates renal tubular cell apoptosis and interstitial fibrosis in obstructive nephropathy. Am J Physiol Renal Physiol 295: F202-F214, 2008.

31. Fudaba Y, Tashiro H, Miyata Y, et al: Oral administration of geranylgeranylacetone protects rat livers from warm ischemic injury. Transplant Proc 31: 2918-2919, 1999.
32. Ikeyama S, Kusumoto K, Miyake H, Rokutan K and Tashiro S: A non-toxic heat shock protein 70 inducer, geranylgeranylacetone, suppresses apoptosis of cultured rat hepatocytes caused by hydrogen peroxide and ethanol. J Hepatol 35: 53-61, 2001.

33. Nishida T, Matsura T, Nakada J, et al: Geranylgeranylacetone protects against acetaminophen-induced hepatotoxicity by inducing heat shock protein 70. Toxicology 219: 187-196, 2006.

34. Kanemura H, Kusumoto K, Miyake H, Tashiro S, Rokutan K and Shimada M: Geranylgeranylacetone prevents acute liver damage after massive hepatectomy in rats through suppression of a CXC chemokine GRO1 and induction of heat shock proteins. J Gastrointest Surg 13: 66-73, 2009.

35. Suzuki S, Maruyama S, Sato W, et al: Geranylgeranylacetone ameliorates ischemic acute renal failure via induction of Hsp70. Kidney Int 67: 2210-2220, 2005.

36. Katsuno M, Sang C, Adachi H, et al: Pharmacological induction of heat-shock proteins alleviates polyglutamine-mediated motor neuron disease. Proc Natl Acad Sci USA 102: 16801-16806, 2005.

37. Ooie T, Kajimoto M, Takahashi N, et al: Effects of insulin resistance on geranylgeranylacetone-induced expression of heat shock protein 72 and cardioprotection in high-fat diet rats. Life Sci 77: 869-881, 2005

38. Kim YH, Song JJ, Kim YC, et al: Geranylgeranylacetone ameliorates acute cochlear damage caused by 3 -nitropropionic acid. Neurotoxicology 31: 317-325, 2010.

39. Zhang K, Zhao T, Huang X, et al: Preinduction of HSP70 promotes hypoxic tolerance and facilitates acclimatization to acute hypobaric hypoxia in mouse brain. Cell Stress Chaperones 14: 407-415, 2009.

40. Hassanzadeh K, Roshangar L, Habibi-asl B, et al: Riluzole prevents morphine-induced apoptosis in rat cerebral cortex. Pharmacol Rep 63: 697-707, 2011.

41. Hassanzadeh K, Habibi-asl B, Farajnia S and Roshangar L: Minocycline prevents morphine-induced apoptosis in rat cerebral cortex and lumbar spinal cord: a possible mechanism for attenuating morphine tolerance. Neurotox Res 19: 649-659, 2011.

42. Crowe AV, Howse M, Bell GM and Henry JA: Substance abuse and the kidney. QJM 93: 147-152, 2000.

43. Singhal PC, Sharma P, Sanwal V, et al: Morphine modulates proliferation of kidney fibroblasts. Kidney Int 53: 350-357, 1998.

44. Singhal PC, Gibbons N and Abramovici M: Long term effects of morphine on mesangial cell proliferation and matrix synthesis. Kidney Int 41: 1560-1570, 1992.

45. Patel J, Manjappa N, Bhat R, Mehrotra P, Bhaskaran M and Singhal PC: Role of oxidative stress and heme oxygenase activity in morphine-induced glomerular epithelial cell growth. Am J Physiol Renal Physiol 285: F861-F869, 2003.

46. Pan J, Zhang Q, Zhang Y, Ouyang Z, Zheng Q and Zheng R: Oxidative stress in heroin administered mice and natural antioxidants protection. Life Sci 77: 183-193, 2005.

47. Singhal PC, Bhaskaran M, Patel J, et al: Role of p38 mitogen-activated protein kinase phosphorylation and Fas-Fas ligand interaction in morphine-induced macrophage apoptosis. J Immunol 168: 4025-4033, 2002.

48. Khanna N and Singh N: Role of caspases in apoptosis and disease. Indian J Physiol Pharmacol 43: 151-159, 1999.

49. Friedlander RM: Apoptosis and caspases in neurodegenerative diseases. N Engl J Med 348: 1365-1375, 2003.

50. Hassanzadeh K, Habibi-asl B, Roshangar L, Nemati M, Ansarin $\mathrm{M}$ and Farajnia S: Intracerebroventricular administration of riluzole prevents morphine-induced apoptosis in the lumbar region of the rat spinal cord. Pharmacol Rep 62: 664-673, 2010

51. Fujita E, Kouroku Y, Jimbo A, Isoai A, Maruyama K and Momoi T: Caspase-12 processing and fragment translocation into nuclei of tunicamycin-treated cells. Cell Death Differ 9: 1108-1114, 2002

52. Nakagawa T, Zhu H, Morishima N, et al: Caspase-12 mediates endoplasmic-reticulum-specific apoptosis and cytotoxicity by amyloid-beta. Nature 403: 98-103, 2000.

53. Calabrese V, Cornelius C, Mancuso C, et al: Vitagenes, dietary antioxidants and neuroprotection in neurodegenerative diseases. Front Biosci 14: 376-397, 2009.

54. Masutani H, Bai J, Kim YC and Yodoi J: Thioredoxin as a neurotrophic cofactor and an important regulator of neuroprotection. Mol Neurobiol 29: 229-242, 2004 
55. Ammon S, Mayer P, Riechert U, Tischmeyer $\mathrm{H}$ and Hollt V: Microarray analysis of genes expressed in the frontal cortex of rats chronically treated with morphine and after naloxone precipitated withdrawal. Brain Res Mol Brain Res 112: 113-125, 2003.

56. Rodriguez Parkitna JM, Bilecki W, Mierzejewski P, et al: Effects of morphine on gene expression in the rat amygdala. J Neurochem 91: 38-48, 2004.

57. Hoshino Y, Nakamura T, Sato A, Mishima M, Yodoi J and Nakamura H: Neurotropin demonstrates cytoprotective effects in lung cells through the induction of thioredoxin-1. Am J Respir Cell Mol Biol 37: 438-446, 2007.

58. Tanito M, Kwon YW, Kondo N, et al: Cytoprotective effects of geranylgeranylacetone against retinal photooxidative damage. J Neurosci 25: 2396-2404, 2005.

59. Tanito M, Masutani H, Kim YC, Nishikawa M, Ohira A and Yodoi J: Sulforaphane induces thioredoxin through the antioxidant-responsive element and attenuates retinal light damage in mice. Invest Ophthalmol Vis Sci 46: 979-987, 2005.
60. Luo FC, Wang SD, Li K, Nakamura H, Yodoi J and Bai J: Panaxatriol saponins extracted from Panax notoginseng induces thioredoxin-1 and prevents 1-methyl-4-phenylpyridinium ioninduced neurotoxicity. J Ethnopharmacol 127: 419-423, 2010.

61. Hashimoto S, Matsumoto K, Gon Y, et al: Thioredoxin negatively regulates p38 MAP kinase activation and IL-6 production by tumor necrosis factor-alpha. Biochem Biophys Res Commun 258: 443-447, 1999.

62. Saitoh M, Nishitoh H, Fujii M, et al: Mammalian thioredoxin is a direct inhibitor of apoptosis signal-regulating kinase (ASK) 1. EMBO J 17: 2596-2606, 1998.

63. Guo S, Wharton W, Moseley P and Shi H: Heat shock protein 70 regulates cellular redox status by modulating glutathione-related enzyme activities. Cell Stress Chaperones 12: 245-254, 2007.

64. Hirota K, Nakamura H, Arai T, et al: Geranylgeranylacetone enhances expression of thioredoxin and suppresses ethanolinduced cytotoxicity in cultured hepatocytes. Biochem Biophys Res Commun 275: 825-830, 2000. 